Article

\title{
Quintessential Inflation with Dynamical Higgs Generation as an Affine Gravity
}

\author{
David Benisty 1,2, Eduardo I. Guendelman ${ }^{1,2,3}$, Emil Nissimov ${ }^{4, *} *$ and Svetlana Pacheva 4 \\ 1 Physics Department, Ben-Gurion University of the Negev, Beer-Sheva 84105, Israel; \\ benidav@post.bgu.ac.il (D.B.); guendelman@fias.uni-frankfurt.de (E.I.G.) \\ 2 Frankfurt Institute for Advanced Studies (FIAS), Ruth-Moufang-Strasse 1, 60438 Frankfurt am Main, Germany \\ 3 Bahamas Advanced Study Institute and Conferences, 4A Ocean Heights, Hill View Circle, Stella Maris, \\ Long Island, Bahamas \\ 4 Institute for Nuclear Research and Nuclear Energy, Bulgarian Academy of Sciences, 1784 Sofia, Bulgaria; \\ svetlanapacheva@gmail.com \\ * Correspondence: emilnissimov6@gmail.com
}

Received: 16 March 2020; Accepted: 13 April 2020; Published: 5 May 2020

\begin{abstract}
First, we propose a scale-invariant modified gravity interacting with a neutral scalar inflaton and a Higgs-like $S U(2) \times U(1)$ iso-doublet scalar field based on the formalism of non-Riemannian (metric-independent) spacetime volume-elements. This model describes, in the physical Einstein frame, a quintessential inflationary scenario driven by the "inflaton" together with the gravity-"inflaton" assisted dynamical spontaneous $S U(2) \times U(1)$ symmetry breaking in the post-inflationary universe, whereas the $S U(2) \times U(1)$ symmetry remains intact in the inflationary epoch. Next, we find the explicit representation of the latter quintessential inflationary model with a dynamical Higgs effect as an Eddington-type purely affine gravity.
\end{abstract}

Keywords: inflation; dark energy; dynamical Higgs effect; quintessence

\section{Introduction}

Studies in cosmology are dominated by the fundamental concept of "inflation" - a period of exponential expansion, which provides a plausible solution for the "puzzles" of the Big-Bang cosmology (the horizon problem, the flatness problem, the magnetic monopole problem, etc.) [1-8]. For more extensive accounts, see the books [9-21]. The most widely discussed mechanism for generating a period of accelerated expansion is through the presence of some vacuum energy. In the context of models with scalar field(s)-driven inflation, vacuum energy density appears naturally when the scalar field(s) acquire an effective potential $U_{\text {eff }}$ which has flat regions so that the scalar field(s) can "slowly roll" [5,6,22-24] and their kinetic energy can be neglected resulting in an energy-momentum tensor of the form $T_{\mu \nu} \simeq-g_{\mu \nu} U_{\text {eff. }}$.

With the discovery of the accelerating expansion of the present universe [25-33], it appears plausible that a small vacuum energy density, usually referred in this case as "dark energy", is also present even today. The two vacuum energy densities, the one of inflation and the other of the dark energy dominated universe nowadays, have however a totally different scale which demands a plausible explanation of how cosmological evolution may naturally interpolate between two apparently quite distinctive physical situations.

The possibility of continuously connecting an inflationary phase of the "early" universe to a slowly accelerating universe of nowadays through the evolution of a single scalar field-the quintessential inflation scenario - has been first studied in [34]. Subsequently, a multitude of different quintessential inflationary models have been proposed: (a) based on modified $f(R)$ gravity [35-37]; (b) based on 
the k-essence concept [38-42]; based on the "variable gravity" model [43]. For an extensive list of references to earlier work on the topic of quintessential inflation, see the References [44-53] (some of them focusing on Higgs inflation) and [54-59], In particular, see the recent Reference [60] for quintessential inflation in the context of Einstein-Gauss-Bonnet gravity and Reference [61] for warm quintessential inflation.

Another parallel groundbreaking development alongside the quintessential inflationary cosmology is the advent of extended modified gravitational theories. The main motivation aims to overcome the limitations of the canonical Einstein's general relativity manifesting themselves in: (i) Cosmology, for solving the problems of dark energy and dark matter and explaining the large scale structure of the Universe [26,62,63]; (ii) Quantum field theory in curved spacetime, due to the non-renormalizabilty of ultraviolet divergences in higher loops [64-69]; and (iii) Modern string theory, given the natural appearance of higher-order curvature invariants and scalar-tensor couplings in low-energy effective field theories [70-74].

Various classes of modified gravity theories have been employed to construct plausible inflationary models: $f(R)$-gravity, scalar-tensor gravity, Gauss-Bonnet gravity (see $[75,76]$ for an extensive review); also recent proposals based on non-local gravity ([77] and references therein) or based on brane-world scenarios ([78] and references therein). Let us recall the first early successful cosmological model based on the extended $f(R)=R+R^{2}$-gravity producing the classical Starobinsky inflationary scalar field potential [2].

For a recent detailed work on quintessential inflation based on $f(R)$-gravity, where the role of dark matter is being played by axions, see Refernece [79].

A broad class of actively developed modified/extended gravitational theories is based on employing (one or more) alternative non-Riemannian spacetime volume-forms, i.e., metric-independent generally covariant volume-elements in the pertinent Lagrangian actions on spacetime manifolds with an ordinary Riemannian geometry, instead of (or alongside with) the canonical Riemannian volume-element $\sqrt{-g} d^{4} x$, whose density is given by the square-root of the determinant of the Riemannian metric $\sqrt{-g} \equiv \sqrt{-\operatorname{det}\left\|g_{\mu v}\right\|}$.

Originally the formalism employing non-Riemannian volume-elements in generally-covariant Lagrangian actions as in Equation (7) below was proposed in [80-84]. The concise geometric formulation was presented in $[85,86]$. A brief outline of the basics of the formalism of non-Riemannian volume-elements is given in Section 2 below.

This formalism was used as a basis for constructing a series of modified gravity-matter models describing unified dark energy and dark matter scenario [87,88], quintessential cosmological models with gravity-assisted and inflaton-assisted dynamical suppression (in the "early" universe) or dynamical generation (in the post-inflationary universe) of electroweak spontaneous symmetry breaking and charge confinement [89-91], as well as a novel mechanism for the supersymmetric Brout-Englert-Higgs effect (dynamical spontaneous supersymmetry breaking) in supergravity [85].

In the present paper our first principal goal is to analyze (Section 3 below) the close interplay between cosmological dynamics and the patterns of (spontaneous) symmetry breaking along the history of universe, which itself is one of the most important paradigms at the interface of particle physics and cosmology. We will extend our construction, started in [89], of a modified gravity model coupled to (the Higgs part) of the standard electroweak matter content (see, for example, [92,93]) besides the scalar "inflaton" field. The main aim here is to provide an explicit realization from first (Lagrangian action) principles of the remarkable proposal of Bekenstein [94] about the so called gravity-assisted dynamical generation of the Higgs effect-dynamical symmetry breaking of the electroweak $S U(2) \times U(1)$ symmetry-without introducing unnatural (according to Bekenstein's opinion) ingredients such as negative ("ghost"-like) mass squared and quartic self-interaction for the Higgs field. Here we study the interrelation between the presence or absence of dynamical spontaneous electroweak symmetry breaking and the different stages of universe's evolution driven 
by the "inflaton"- triggering inflation in the "early" universe as well as representing quintessential variable dark-energy in the "late" universe.

It is shown that during inflation there is no spontaneous electroweak symmetry breaking and the Higgs field resides in its "wrong" vacuum state ("wrong" from the point of view of standard high-energy particle physics). The non-trivial symmetry-breaking Higgs vacuum is dynamically generated in the post-inflationary epoch.

Let us specifically stress that this mechanism is different from the widely discussed scenario of Higgs inflation, where the Higgs field triggers the inflation in the "early" universe through a non-minimal coupling to gravity [95-110]. In our scenario the impact of the Higgs field dynamics starts after end of inflation.

Another ground-laying branch of gravitational theories is the purely affine gravity formalism, first proposed in [111-115]. It has attracted since then a significant interest primarily due to the established dynamical equivalence [116] of the three principal formulations of standard Einstein's gravity: purely metric (second-order formalism), metric-affine (Palatini or first-order formalism) and purely affine formalism. For more recent developments and list of references, see [117-134], in particular about incorporating torsion and explaining dark energy as an instrinsic property of space-time.

To establish the connection of our non-Riemannian volume-element formalism and the purely affine formalism, our next task (Section 4) will be to represent the above quintessential inflationary model with a dynamical Higgs effect in the form of a no-metric purely affine (Eddington-type) gravity.

\section{The Essence of the Non-Riemannian Volume-Form Formalism}

Volume-forms define volume-elements (generally covariant integration measures) over differentiable manifolds $\mathcal{M}$, not necessarily Riemannian ones, so no metric is a priori needed [135]. They are given by nonsingular maximal-rank differential forms $\omega$ on $\mathcal{M}$ (for definiteness we will consider the case of $D=4$ dimensional $\mathcal{M}$ ):

$$
\int_{\mathcal{M}} \omega(\ldots)=\int_{\mathcal{M}} d^{4} x \Omega(\ldots)
$$

where:

$$
\omega=\frac{1}{4 !} \omega_{\mu v \kappa \lambda} d x^{\mu} \wedge d x^{v} \wedge d x^{\kappa} \wedge d x^{\lambda}, \quad \omega_{\mu v \kappa \lambda}=-\varepsilon_{\mu v \kappa \lambda} \Omega, \Omega=\frac{1}{4 !} \varepsilon^{\mu v \kappa \lambda} \omega_{\mu v \kappa \lambda} .
$$

The conventions for the alternating symbols $\varepsilon^{\mu \nu \kappa \lambda}$ and $\varepsilon_{\mu \nu \kappa \lambda}$ are: $\varepsilon^{0123}=1$ and $\varepsilon_{0123}=-1$.

The volume-element density (integration measure density) $\Omega$ transforms as scalar density under general coordinate reparametrizations.

In standard general-relativistic theories the Riemannian spacetime volume-form is defined through the tetrad canonical one-forms $e^{A}=e_{\mu}^{A} d x^{\mu}(A=0,1,2,3)$ :

$$
\omega=e^{0} \wedge e^{1} \wedge e^{2} \wedge e^{3}=\operatorname{det}\left\|e_{\mu}^{A}\right\| d x^{0} \wedge d x^{1} \wedge d x^{2} \wedge d x^{3},
$$

which yields:

$$
\Omega=\operatorname{det}\left\|e_{\mu}^{A}\right\|=\sqrt{-\operatorname{det}\left\|g_{\mu \nu}\right\|} \equiv \sqrt{-g} .
$$

Instead of $\sqrt{-g} d^{4} x$ we can employ another alternative non-Riemannian volume-element as in (1) and (2) given by a non-singular exact 4 -form $\omega=d A$ where:

$$
\left.A=\frac{1}{3 !} A_{\mu v \kappa} d x^{\mu} \wedge d x^{\nu} \wedge d x^{\kappa} \quad \longrightarrow \quad \omega=\frac{1}{4 !} \partial_{[\mu} A_{v \kappa} \lambda\right] d x^{\mu} \wedge d x^{v} \wedge d x^{\kappa} \wedge d x^{\lambda} .
$$


Therefore, the corresponding non-Riemannian volume-element density

$$
\Omega \equiv \Phi(A)=\frac{1}{3 !} \varepsilon^{\mu v \kappa \lambda} \partial_{\mu} A_{\nu \kappa \lambda} .
$$

is defined in terms of the dual field-strength scalar density of an auxiliary rank 3 tensor gauge field $A_{\mu v \kappa}$.

In the next Section we will discuss in some detail the properties of a quintessential inflationary model coupled to a truncated version of the electro-weak particle content carrying the standard electro-weak $S U(2) \times U(1)$ symmetry. Namely, for simplicity we retain only a Higgs-like scalar field and discard the electro-weak gauge fields and fermions.

Before proceeding let us note the following important property of Lagrangian action terms involving (one or more) non-Riemannian volume-elements:

$$
S=\int d^{4} x \sum_{j} \Phi\left(A^{(j)}\right) \mathcal{L}^{(j)}(\text { other fields })+\ldots
$$

The equations of motion of (7) with respect to the auxiliary tensor gauge fields $A_{\mu \nu \kappa}^{(j)}$ according to (6) imply:

$$
\left.\left.\partial_{\mu} \mathcal{L}^{(j)} \text { (other fields }\right)=0 \longrightarrow \mathcal{L}^{(j)} \text { (other fields }\right)=M_{j},
$$

where $M_{j}$ are free integration constants not present in the original action (7). This illustrates the significant advantage of the non-Riemannian volume-element formalism over the "Lagrange-multiplier gravity" method [136], which appeared a decade later and which requires picking a priori some ad hoc constant as opposed to the dynamical appearance of the arbitrary integration constants (8). For further advantages of the non-Riemannian volume-element formalism, see the above remarks.

A characteristic feature of the modified gravitational theories (7) is that when starting in the first-order (Palatini) formalism, all non-Riemannian volume-elements $\Phi\left(A^{(j)}\right)$ yield almost pure-gauge degrees of freedom, i.e., they do not introduce any additional physical (field-propagating) gravitational degrees of freedom except for few discrete degrees of freedom with conserved canonical momenta appearing as arbitrary integration constants $M_{j}$. The reason is that the modified gravity action (7) in Palatini formalism is linear with respect to the velocities of some of the components of the auxiliary gauge fields $A_{\mu v \kappa}^{(j)}$ defining the non-Riemannian volume-element densities, and does not depend on the velocities of the rest of auxiliary gauge field components. The (almost) pure-gauge nature of the latter is explicitly shown in $[86,89]$ (Appendix A) employing the standard canonical Hamiltonian treatment of systems with gauge symmetries, i.e., systems with first-class Hamiltonian constraints according to the classification of Dirac $[137,138]$.

\section{Quintessential Inflationary Model with Dynamical Higgs Effect}

Our starting point is the following specific example of the general class of modified gravity models $[85,86,89-91,139,140])$ involving several non-Riemannian volume-elements (using units with $\left.16 \pi G_{\text {Newton }}=1\right)$ :

$$
S=\int d^{4} x \Phi_{1}(A)\left[R(g, \Gamma)-2 \Lambda_{0} \frac{\Phi_{1}(A)}{\sqrt{-g}}+X_{\phi}+f_{1} e^{\alpha \phi}+X_{\sigma}-V_{0}(\sigma) e^{\alpha \phi}\right]+\int d^{4} x \Phi_{2}(B)\left[f_{2} e^{2 \alpha \phi}-\frac{\Phi_{0}(C)}{\sqrt{-g}}\right] .
$$

Here the following notations are used:

- The scalar curvature $R(g, \Gamma)=g^{\mu v} R_{\mu v}(\Gamma)$ is given in terms of the Ricci tensor $R_{\mu v}(\Gamma)$ in the first-order (Palatini) formalism:

$$
R_{\mu v}(\Gamma)=\partial_{\alpha} \Gamma_{\mu v}^{\alpha}-\partial_{v} \Gamma_{\mu \alpha}^{\alpha}+\Gamma_{\alpha \beta}^{\alpha} \Gamma_{\mu v}^{\beta}-\Gamma_{\beta v}^{\alpha} \Gamma_{\mu \alpha}^{\beta}
$$


defined by the affine connection $\Gamma_{\mu \nu}^{\lambda}$ a priori independent of the metric $g_{\mu v}$.

- The non-Riemannian volume-element densities $\Phi_{1}(A), \Phi_{2}(B), \Phi_{0}(C)$ are defined as in (6):

$$
\Phi_{1}(A)=\frac{1}{3 !} \varepsilon^{\mu v \kappa \lambda} \partial_{\mu} A_{v \kappa \lambda}, \quad \Phi_{2}(B)=\frac{1}{3 !} \varepsilon^{\mu \nu \kappa \lambda} \partial_{\mu} B_{v \kappa \lambda}, \quad \Phi_{0}(C)=\frac{1}{3 !} \varepsilon^{\mu v \kappa \lambda} \partial_{\mu} C_{v \kappa \lambda} .
$$

- $\phi$ is a neutral scalar "inflaton" and $\sigma \equiv\left(\sigma_{a}\right)$ is a complex $S U(2) \times U(1)$ iso-doublet Higgs-like scalar field with the isospinor index $a=+, 0$ indicating the corresponding $U(1)$ charge. The corresponding kinetic energy terms in (9) read:

$$
X_{\phi} \equiv-\frac{1}{2} g^{\mu v} \partial_{\mu} \phi \partial_{\nu} \phi, \quad X_{\sigma} \equiv-g^{\mu v} \partial_{\mu} \sigma_{a}^{*} \partial_{\nu} \sigma_{a},
$$

and

$$
V_{0}(\sigma) \equiv m_{0}^{2} \sigma_{a}^{*} \sigma_{a},
$$

is a canonical mass term for the Higgs-like field, i.e., neither negative ("ghost-like") mass-squared term nor quartic self-interaction are introduced unlike the case in the standard electro-weak model [92,93].

- $f_{1,2}$ and $\alpha$ are dimensionful coupling constants in the "inflaton" potential. The $\Lambda_{0}$ is a small dimensional constant which will be identified in the sequel with the "late" universe cosmological constant in the dark energy dominated accelerated expansion's epoch.

The specific form of the action (9) is fixed by the requirement of global Weyl-scale invariance under:

$$
\begin{aligned}
g_{\mu \nu} \rightarrow \lambda g_{\mu v}, A_{\mu v \kappa} \rightarrow \lambda A_{\mu v \kappa}, B_{\mu v \kappa} & \rightarrow \lambda^{2} B_{\mu v \kappa}, C_{\mu v \kappa} \rightarrow C_{\mu v \kappa} \\
\phi & \rightarrow \phi-\frac{1}{\alpha} \ln \lambda, \quad \sigma_{a} \rightarrow \sigma_{a},
\end{aligned}
$$

where the scaling parameter is $\lambda=$ const. The importance of global scale symmetry within the context of non-Riemannian volume-element formalism has been already stressed in the first original papers (see [82]), where in particular models with spontaneously broken dilatation symmetry have been constructed along these lines, which are free of the Fifth Force Problem [84].

Varying the action (9) with respect to $g^{\mu \nu}, \Gamma_{\mu v}^{\lambda}, A_{\mu \nu \lambda}, B_{\mu \nu \lambda}, C_{\mu v \lambda}, \phi$ and $\sigma^{a}$, yield the following equations of motion, respectively:

$$
\begin{array}{r}
R_{\mu v}(\Gamma)-\Lambda_{0} \frac{\Phi_{1}(A)}{\sqrt{-g}} g_{\mu v}-\frac{1}{2} \partial_{\mu} \phi \partial_{\nu} \phi-\partial_{\mu} \sigma_{a}^{*} \partial_{\nu} \sigma_{a}-\frac{1}{2} g_{\mu \nu} \frac{\Phi_{2}(B) \Phi_{0}(C)}{\Phi_{1}(A) \sqrt{-g}}=0, \\
\Phi_{1}(A) g^{\mu v}\left(\nabla_{\lambda} \delta \Gamma_{\mu \nu}^{\lambda}-\nabla_{\mu} \delta \Gamma_{\lambda \nu}^{\lambda}\right)=0, \\
g^{\mu \nu}\left(R_{\mu v}(\Gamma)-\frac{1}{2} \partial_{\mu} \phi \partial_{\nu} \phi-\partial_{\mu} \sigma_{a}^{*} \partial_{\nu} \sigma_{a}\right)-4 \Lambda_{0} \frac{\Phi_{1}(A)}{\sqrt{-g}}+\left(f_{1}-m_{0}^{2} \sigma_{a}^{*} \sigma_{a}\right) e^{\alpha \phi}=M_{1} \equiv \text { const }, \\
f_{2} e^{-2 \alpha \phi}-\frac{\Phi_{0}(C)}{\sqrt{-g}}=-M_{2} \equiv \text { const }, \frac{\Phi_{2}(B)}{\sqrt{-g}}=\chi_{2} \equiv \text { const }, \\
\partial_{\mu}\left(\Phi_{1}(A) g^{\mu v} \partial_{\nu} \phi\right)+\alpha \Phi_{1}(A)\left(f_{1}-m_{0}^{2} \sigma_{a}^{*} \sigma_{a}\right) e^{\alpha \phi}+2 \alpha \Phi_{2}(B) f_{2} e^{2 \alpha \phi}=0, \\
\partial_{\mu}\left(\Phi_{1}(A) g^{\mu v} \partial_{\nu} \sigma_{a}\right)-\Phi_{1}(A) m_{0}^{2} e^{\alpha \phi} \sigma_{a}=0 .
\end{array}
$$

Equations (18) and (19) are special cases of the general Equation (8) discussed above. Here $M_{1,2}$ and $\chi_{2}$ are arbitrary (dimensional and dimensionless, respectively) integration constants, with $M_{1,2}$ triggering a spontaneous breaking of the global Weyl-scale symmetry (15). 
Taking the trace of Equation (16) and comparing with Equations (18) and (19), we find for the ratio of volume-element densities:

$$
\chi_{1} \equiv \frac{\Phi_{1}(A)}{\sqrt{-g}}=\frac{2 \chi_{2}\left(f_{2} e^{2 \alpha \phi}+M_{2}\right)}{M_{1}+\left(m_{0}^{2} \sigma_{a}^{*} \sigma_{a}-f_{1}\right) e^{\alpha \phi}} \equiv \chi_{1}(\phi, \sigma) .
$$

On the other hand, following analogous derivation in [82], Equation (17) yields a solution for $\Gamma_{\nu \lambda}^{\mu}$ as a Levi-Civita connection:

$$
\Gamma_{\nu \lambda}^{\mu}=\Gamma_{\nu \lambda}^{\mu}(\bar{g})=\frac{1}{2} \bar{g}^{\mu \kappa}\left(\partial_{\nu} \bar{g}_{\lambda \kappa}+\partial_{\lambda} \bar{g}_{v \kappa}-\partial_{\kappa} \bar{g}_{v \lambda}\right)
$$

with respect to a Weyl-conformally rescaled metric:

$$
\bar{g}_{\mu v}=\chi_{1}(\phi, \sigma) g_{\mu v}
$$

with $\chi_{1}(\phi, \sigma)$ as in (22).

Conformal transformation $g_{\mu \nu} \rightarrow \bar{g}_{\mu v}$ via (24) convert the modified gravity action (9) into the physical Einstein-frame action (objects in the Einstein-frame indicated by a bar):

$$
S_{\mathrm{EF}}=\int d^{4} x \sqrt{-\bar{g}}\left[R(\bar{g})-\frac{1}{2} \bar{g}^{\mu v} \partial_{\mu} \phi \partial_{\nu} \phi-\bar{g}^{\mu v} \partial_{\mu} \sigma_{a}^{*} \partial_{\nu} \sigma_{a}-U_{\mathrm{eff}}(\phi, \sigma)\right],
$$

with an effective Einstein-frame scalar field potential:

$$
\begin{array}{r}
U_{\mathrm{eff}}(\phi, \sigma) \equiv \frac{M_{1}+e^{\alpha \phi}\left(m_{0}^{2} \sigma_{a}^{*} \sigma_{a}-f_{1}\right)}{\chi_{1}(\phi, \sigma)}-\frac{\chi_{2}\left(f_{2} e^{2 \alpha \phi}+M_{2}\right)}{\left(\chi_{1}(\phi, \sigma)\right)^{2}}+2 \Lambda_{0} \\
=\frac{\left[M_{1}+e^{\alpha \phi}\left(m_{0}^{2} \sigma_{a}^{*} \sigma_{a}-f_{1}\right)\right]^{2}}{4 \chi_{2}\left(f_{2} e^{2 \alpha \phi}+M_{2}\right)}+2 \Lambda_{0},
\end{array}
$$

which is entirely dynamically generated due to the appearance of the free integration constants $M_{1,2}$ and $\chi_{2}$ (18) and (19).

As discussed in $[86,90,91,141]$ the scalar potential $U_{\text {eff }}(\phi, \sigma)(26)$ has a remarkable feature: it possesses two (infinitely) large flat regions as a function of $\phi$ at $\sigma_{a}=$ fixed (see the graphical representation on Figure 1) with the following properties:

- (a) (-) flat "inflaton" region for large negative values of $\phi$ (and $\sigma_{a}$ is finite) corresponding to the "slow-roll" inflationary evolution of the "early" universe driven by $\phi$. Here the effective potential (26) reduces to (an almost) constant value independent of the finite value of $\sigma_{a}$ 一this is energy scale of the inflationary epoch:

$$
U_{\mathrm{eff}}(\phi, \sigma) \simeq U_{(-)}=\frac{M_{1}^{2}}{4 \chi_{2} M_{2}}+2 \Lambda_{0} .
$$

Thus, in the "early" universe the Higgs-like field $\sigma_{a}$ must be (approximately) either massless or constant with no non-zero vacuum expectation value, therefore there is no spontaneous breaking of $S U(2) \times U(1)$ symmetry. Moreover, in fact as shown in the Remark below, $\sigma_{a}$ does not participate in the "slow-roll" inflationary evolution, so $\sigma$ stays constant there equal to the "false"vacuum value $\sigma=0$. 
- (b) (+) flat "inflaton" region for large positive values of $\phi$ (and $\sigma_{a}$-finite) corresponding to the evolution of the post-inflationary ("late") universe, where:

$$
U_{\text {eff }}(\phi, \sigma) \simeq U_{(+)}(\sigma)=\frac{\left(m_{0}^{2} \sigma_{a}^{*} \sigma_{a}-f_{1}\right)^{2}}{4 \chi_{2} f_{2}}+2 \Lambda_{0}
$$

acquires the form of a dynamically induced $S U(2) \times U(1)$ spontaneous symmetry breaking Higgs potential with a Higgs "vacuum" at:

$$
\left|\sigma_{\mathrm{vac}}\right|=\frac{1}{m_{0}} \sqrt{f_{1}},
$$

where the parameters are naturally identified as:

$$
f_{1} \sim M_{E W}^{4}, \quad m_{0} \sim M_{E W}
$$

in terms of the electro-weak energy scale $M_{E W} \sim 10^{-16} M_{P l}$.

- Thus, the residual cosmological constant $\Lambda_{0}$ in (28) has to be identified with the current epoch observable cosmological constant $\left(\sim 10^{-122} M_{P l}^{4}\right)$ and, therefore, according to (27) the integration constants $M_{1,2}$ are naturally identified by orders of magnitude as

$$
M_{1} \sim M_{2} \sim 10^{-8} M_{P l}^{4},
$$

since in the latter case the order of magnitude of the vacuum energy density in the (-) flat region (27) becomes:

$$
U_{(-)} \sim M_{1}^{2} / M_{2} \sim 10^{-8} M_{P l}^{4},
$$

which conforms to the Planck Collaboration data $[142,143]$ for the "early" universe's energy scale of inflation being of order $10^{-2} M_{P l}$.

- Here the order of magnitude for $f_{2}$ is determined from the mass term of the Higgs-like field $\sigma$ in the $(+)$ flat region resulting from (28) upon expansion around the Higgs vacuum $\left(\sigma=\sigma_{\mathrm{vac}}+\widetilde{\sigma}\right)$ :

$$
\frac{f_{1} m_{0}^{2}}{\chi_{2} f_{2}}\left(\widetilde{\sigma}_{a}\right)^{*}\left(\widetilde{\sigma}_{a}\right),
$$

which implies that:

$$
f_{2} \sim f_{1} \sim M_{E W}^{4} .
$$

- Let us specifically note that the viability of the present model (in a slightly simplified form without the Higgs scalar) concerning confrontation with the observational data has already been analyzed and confirmed numerically in Reference [141]. In particular, a graphical plot of the evolution of $r$ (tensor-to-scalar ratio) vs. $n_{S}$ (scalar spectral index) has been provided there. 


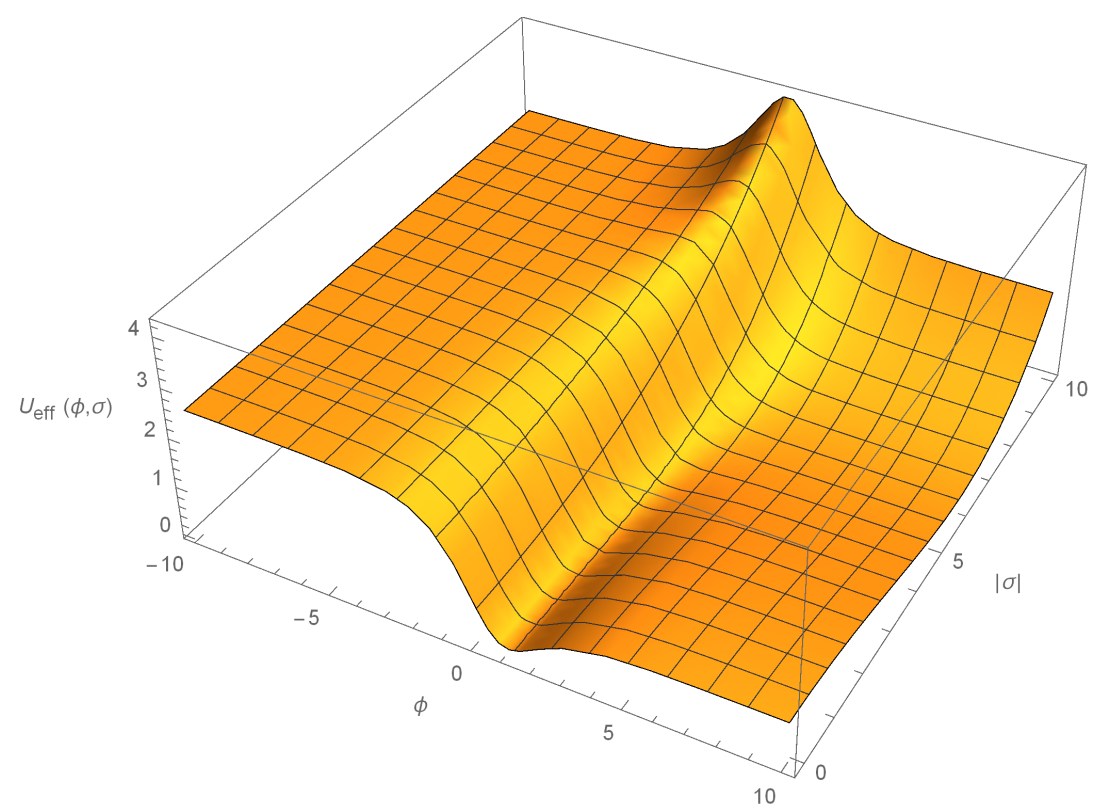

Figure 1. Qualitative shape of the two-dimensional plot for the effective scalar potential $U_{\text {eff }}(\phi, \sigma)(26)$.

Remark 1. Assuming that in the (-) flat "inflaton" region (for large negative values of $\phi$ and $\sigma_{a}$-finite) both the "inflaton" $\phi$ and the Higgs-like field $\sigma_{a}$ evolve in a "slow-role" regime, their "slow-role" equations of motion in the standard FLRW (Friedmann-Lemaitre-Robertson-Walker) reduction of the Einstein-frame metric $\left(\bar{g}_{\mu \nu} d x^{\mu} d x^{v} \equiv-N^{2}(t) d t^{2}+a^{2}(t) d \vec{x} . d \vec{x}\right)$ read accordingly (see, for example, $\left.[5,6,22,23]\right)$ :

$$
\begin{array}{r}
\dot{\phi} \simeq-\frac{1}{3 H} \frac{\partial U_{\mathrm{eff}}(\phi, \sigma)}{\partial \phi}, \quad \frac{\partial U_{\mathrm{eff}}(\phi, \sigma)}{\partial \phi}=\frac{\alpha e^{\alpha \phi}\left[M_{1}+e^{\alpha \phi}\left(m_{0}^{2}|\sigma|^{2}-f_{1}\right)\right]\left[M_{2}\left(m_{0}^{2}|\sigma|^{2}-f_{1}\right)-M_{1} f_{2} e^{\alpha \phi}\right]}{2 \chi_{2}\left(M_{2}+f_{2} e^{2 \alpha \phi}\right)^{2}} \\
\dot{\sigma} \simeq-\frac{1}{3 H} \frac{\partial U_{\mathrm{eff}}(\phi, \sigma)}{\partial \sigma} \longrightarrow \frac{d|\sigma|}{d t} \simeq-\frac{1}{3 H} \frac{m_{0}^{2}|\sigma| e^{\alpha \phi}\left[M_{1}+e^{\alpha \phi}\left(m_{0}^{2}|\sigma|^{2}-f_{1}\right)\right]}{2 \chi_{2}\left(M_{2}+f_{2} e^{2 \alpha \phi}\right)},
\end{array}
$$

where $|\sigma|^{2} \equiv \sigma_{a}^{*} \sigma_{a}$ and $H=\frac{\dot{a}}{a}$ denotes the Hubble parameter. Equations (35) and (36) define parametrically a curve $|\sigma|=|\sigma|(\phi)$ in the two-field $(\phi,|\sigma|)$ target space. Equivalently, this curve is defined through the differential equation:

$$
\frac{d|\sigma|}{d z} \simeq \frac{m_{0}^{2}|\sigma(z)|\left(M_{2}+f_{2} z^{2}\right)}{\alpha^{2} z\left[M_{2}\left(m_{0}^{2}|\sigma(z)|^{2}-f_{1}\right)-M_{1} f_{2} z\right]} \quad, \quad z \equiv e^{\alpha \phi} .
$$

In the (-) flat "inflaton" region ( $\phi$-large negative) $z$ is very small, so in this case Equation(37) can be rewritten as:

$$
\alpha\left(|\sigma|-\frac{f_{1}}{m_{0}^{2}|\sigma|}\right) d|\sigma| \simeq \frac{d z}{z} \quad \longrightarrow \quad \alpha\left(\frac{1}{2}|\sigma(z)|^{2}-\frac{f_{1}}{m_{0}^{2}} \ln |\sigma(z)|\right) \simeq \ln z
$$

Obviously, a consistent solution $|\sigma(z)|$ of (38) does not exist for $z=e^{\alpha \phi} \rightarrow 0$, therefore, the assumption for the "slow-roll" evolution (36) of the Higgs-like field $\sigma_{a}$ in the inflationary region (large negative values of $\phi$ ) is invalid. Thus $|\sigma|$ must be constant and Equation (36) implies $|\sigma|=0$ in the (-) flat "inflaton" region.

To conclude this section, we see that thanks to the remarkable dynamically generated scalar potential (26) the "inflaton" $\phi$ plays the role both of driving "slow-roll" inflationary dynamics in the "early" universe, as well as it plays the role of a quintessential variable dark-energy field triggering slowly accelerating de Sitter expansion in the "late" universe. 
Accordingly, gravity-inflaton dynamics generates dynamically spontaneous $S U(2) \times U(1)$ symmetry breaking - Higgs effect - in the post-inflationary epoch, whereas it dynamically suppresses spontaneous symmetry breaking during inflation in the "early" universe. Namely, the dynamical transition from the "false" Higgs vacuum to the genuine electroweak spontaneously broken vacuum is driven by the inflaton evolving from (large) negative values (on the " $(-)$ " flat region (27) of the scalar potential (26)) to (large) positive values (on the " $(+)$ " flat region (28) of the scalar potential (26)).

Thus, our scale invariant modified gravity model (9) in its Einstein-frame representation (25) and (26) turns out to be an explicit implementation of Bekenstein's idea [94] about a gravity-assisted spontaneous symmetry breaking of electro-weak (Higgs) type without invoking negative mass squared and a quartic Higgs field self-interaction unlike the canonical case in the standard particle model $[92,93]$.

\section{Eddinton-Type No-Metric Gravity and Quintessential Inflation}

Let us now consider a generic model of gravity, with some Riemannian metric $\bar{g}_{\mu v}$ and with the ordinary Riemannian volume-element $\sqrt{-\bar{g}}$ within the first-order (Palatini) formalism, interacting with a multi-component scalar field $\phi^{A}, A=1, \ldots, N$ (using again units with $16 \pi G_{\text {Newton }}=1$ ):

$$
S=\int d^{4} x \sqrt{-\bar{g}}\left[\bar{g}^{\mu v} R_{\mu v}(\Gamma)-\frac{1}{2} \bar{g}^{\mu v} h_{A B} \partial_{\mu} \phi^{A} \partial_{\nu} \phi^{B}-U(\phi)\right],
$$

where the Ricci tensor $R_{\mu v}(\Gamma)$ is the same as in (10), and $h_{A B}(\phi)$ indicates some "metric" in the scalar field target space (in the present case it will be just a unit matrix).

The equations of motion with respect to $g^{\mu v}, \phi^{A}$ and $\Gamma_{\mu v}^{\lambda}$ read accordingly:

$$
\begin{array}{r}
R_{\mu \nu}(\Gamma)=\frac{1}{2}\left(T_{\mu \nu}-\frac{1}{2} \bar{g}_{\mu \nu} T_{\lambda}^{\lambda}\right), \\
T_{\mu \nu}=h_{A B}(\phi) \partial_{\mu} \phi^{A} \partial_{\nu} \phi^{B}-\bar{g}_{\mu \nu}\left[\frac{1}{2} \bar{g}^{\kappa \lambda} h_{A B}(\phi) \partial_{\kappa} \phi^{A} \partial_{\lambda} \phi^{B}+U(\phi)\right], \\
\frac{1}{\sqrt{-\bar{g}}} \partial_{\mu}\left(\sqrt{-\bar{g}} g^{\mu \nu} h_{A B} \partial_{\nu} \phi^{B}\right)-\frac{1}{2} \bar{g}^{\mu \nu} \frac{\partial h_{C D}}{\partial \phi^{A}} \partial_{\mu} \phi^{C} \partial_{\nu} \phi^{D}-\frac{\partial U(\phi)}{\partial \phi^{a}}=0, \\
\int d^{4} x \sqrt{-\bar{g}} \bar{g}^{\mu \nu}\left(\nabla_{\lambda} \delta \Gamma_{\mu \nu}^{\lambda}-\nabla_{\mu} \delta \Gamma_{\lambda \nu}^{\lambda}\right)=0
\end{array}
$$

Following again the analogous derivation in [82], the solution of Equation (43) is that $\Gamma_{\mu \nu}^{\lambda}$ becomes the canonical Levi-Civita connection w.r.t. $\bar{g}_{\mu v}$ :

$$
\Gamma_{v \lambda}^{\mu}=\Gamma_{v \lambda}^{\mu}(\bar{g})=\frac{1}{2} \bar{g}^{\mu \kappa}\left(\partial_{\nu} \bar{g}_{\lambda \kappa}+\partial_{\lambda} \bar{g}_{\nu \kappa}-\partial_{\kappa} \bar{g}_{\nu \lambda}\right)
$$

Equations (40) and (41) can be equivalently written as:

$$
\bar{g}_{\mu \nu}=\frac{2}{U(\phi)}\left(R_{\mu v}(\Gamma)-\frac{1}{2} h_{A B}(\phi) \partial_{\mu} \phi^{A} \partial_{\nu} \phi^{B}\right),
$$

that is, the metric $\bar{g}_{\mu \nu}$ in (39) is expressed entirely in terms of the affine connection and the matter field.

Now, we will show that the gravity-matter theory (39) is equivalent, in a sense of producing the same equations of motion (41)-(44), to the following Eddington-type purely affine gravity theory:

$$
S_{E d d}=\int d^{4} x \frac{2}{U(\phi)} \sqrt{\operatorname{det}\left\|R_{\mu \nu}(\Gamma)-\frac{1}{2} h_{A B}(\phi) \partial_{\mu} \phi^{A} \partial_{\nu} \phi^{B}\right\|}
$$

i.e., (46) does not involve at all a Riemannian metric. 
Indeed, varying the action (46) w.r.t. $\Gamma_{\mu \nu}^{\lambda}$ and $\phi^{a}$ we get:

$$
\frac{2}{U(\phi)} \sqrt{\operatorname{det}\left\|H_{\alpha \beta}(\Gamma, \phi, \sigma)\right\|}\left(H^{-1}((\Gamma, \phi, \sigma))^{\mu v}\left(\nabla_{\lambda} \delta \Gamma_{\mu v}^{\lambda}-\nabla_{\mu} \delta \Gamma_{\lambda v}^{\lambda}\right)=0,\right.
$$

with the short-hand notation:

$$
H_{\mu v}(\Gamma, \phi) \equiv R_{\mu v}(\Gamma)-\frac{1}{2} h_{A B}(\phi) \partial_{\mu} \phi^{A} \partial_{\nu} \phi^{B},
$$

and

$$
\begin{array}{r}
\partial_{\mu}\left(\frac{1}{U(\phi)} \sqrt{\left.\operatorname{det}\left\|H_{\alpha \beta}(\Gamma, \phi)\right\|\left(H^{-1}(\Gamma, \phi)\right)^{\mu v} h_{A B}(\phi) \partial_{\nu} \phi^{B}\right)}\right. \\
-\frac{1}{2 U(\phi)} \sqrt{\operatorname{det}\left\|H_{\alpha \beta}(\Gamma, \phi)\right\|}\left(H^{-1}(\Gamma, \phi)\right)^{\mu \nu} \frac{\partial h_{C D}}{\partial \phi^{A}} \partial_{\mu} \phi^{C} \partial_{\nu} \phi^{D}+2 \frac{\partial}{\partial \phi}\left(\frac{1}{U(\phi)}\right) \sqrt{\operatorname{det}\left\|H_{\alpha \beta}(\Gamma, \phi)\right\|}=0 .
\end{array}
$$

Now, using the identification Equation (45) for the Riemannian metric $\bar{g}_{\mu v}=\frac{2}{U(\phi)} H_{\mu v}(\Gamma, \phi)$ with $H_{\mu v}(\Gamma, \phi)$ as in (48), Equations (47)-(49) become identical to Equations (42) and (43), respectively.

The above derivation of purely affine gravity interacting with multi-component scalar fields appeared previously in [144]. Historically, this formulation was proposed for the first time in [115] in the special case of a single Klein-Gordon field with $U(\phi)=\frac{1}{2} m^{2} \phi^{2}$, see also [145].

Applying the above established equivalence between the models (39) and (46) to the initial modified gravity action (9) and its Einstein-frame representation (25) with $U_{\text {eff }}(\phi, \sigma)$ as in (26), analyzed in Section 3 above, we find that the following specific Eddington-type purely affine no-metric gravity model:

$$
\begin{array}{r}
S_{E d d}=\int d^{4} x \frac{8 \chi_{2}\left(f_{2} e^{-2 \alpha \phi}+M_{2}\right)}{\left[M_{1}+\left(m_{0}^{2} \sigma_{a}^{*} \sigma_{a}-f_{1} e^{-\alpha \phi}\right)\right]^{2}+8 \Lambda_{0} \chi_{2}\left(f_{2} e^{-2 \alpha \phi}+M_{2}\right)} \\
\times \sqrt{\operatorname{det}\left\|R_{\mu v}(\Gamma)-\frac{1}{2} \partial_{\mu} \phi \partial_{\nu} \phi-\partial_{\mu} \sigma_{a}^{*} \partial_{\nu} \sigma_{a}\right\|},
\end{array}
$$

actually describes a quintessential inflationary dynamics with dynamically generated Higgs effect in the post-iflationary epoch with all the properties discussed in Section 3. The metric $g_{\mu \nu}$ in the initial modified scale-invariant gravity action (9) with non-Riemannian volume-elements, taking into account relation (45) applied for the Einstein-frame metric (24) where $U(\phi)=U_{\text {eff }}(\phi, \sigma)$ (26) and using the on-shell relations (19) and (22), is identified as:

$$
g_{\mu v}=\frac{2}{\chi_{1}(\phi, \sigma) U_{\mathrm{eff}}(\phi, \sigma)}\left[R_{\mu v}(\Gamma)-\frac{1}{2} \partial_{\mu} \phi \partial_{\nu} \phi-\partial_{\mu} \sigma_{a}^{*} \partial_{\nu} \sigma_{a}\right],
$$

with $R_{\mu v}(\Gamma)$ as in (10), and $\chi_{1}(\phi, \sigma)$ and $U_{\text {eff }}(\phi, \sigma)$ explicitly given in (22) and (26), respectively:

$$
\frac{1}{\chi_{1}(\phi, \sigma) U_{\mathrm{eff}}(\phi, \sigma)}=\frac{2\left[M_{1}+e^{\alpha \phi}\left(m_{0}^{2} \sigma_{a}^{*} \sigma_{a}-f_{1}\right)\right]}{\left[M_{1}+e^{\alpha \phi}\left(m_{0}^{2} \sigma_{a}^{*} \sigma_{a}-f_{1}\right)\right]^{2}+8 \Lambda_{0} \chi_{2}\left(f_{2} e^{2 \alpha \phi}+M_{2}\right)} .
$$

\section{Conclusions}

In the present paper we have employed two fundamental concepts, namely, non-Riemannian metric-independent spacetime volume-elements and (global) scale invariance, to construct a self-consistent model of modified gravity coupled to a neutral scalar "inflaton" and to a Higgs-like $S U(2) \times U(1)$ iso-boublet scalar possessing the following extraordinary features: 
(a) In the physical Einstein frame, thanks to a dynamical generation of a remarkable scalar potential with two long flat "inflaton" regions with vastly different heights, the model describes a plausible quintessential inflationary scenario, driven by the "inflaton", with a "slow-roll" inflationary stage in the "early" universe and a slow accelerating de Sitter expansion in the "late" universe;

(b) This model provides an explanation of the interplay between cosmological dynamics and the patterns of symmetry breaking during the evolution of the universe. Namely, we find an explicit realization from first (Lagrangian-action) principles of the noteworthy proposal of Bekenstein from 1986 about "gravity-assisted" dynamical Higgs-like spontaneous symmetry breakdown (Higgs effect). We exhibit gravity-"inflaton" suppression of the Higgs effect during inflation, i.e., no electroweak spontaneous breakdown there), whereas in the post-inflationary epoch a Higgs-type symmetry breaking potential is dynamically created.

(c) The coupling constants in the initial modified gravity action are naturally identified as powers of the standard electroweak mass scale.

(d) It is shown how to represent the above quintessential inflationary model with a dynamical Higgs effect in the form of a no-metric purely affine (Eddington-type) gravity.

A next important task is to study in some detail, within the present quintessential inflationary scenario with a dynamical Higgs effect, the numerical solutions for the basic inflationary observables (scalar power spectral index, tensor-to-scalar ratio, etc.) extending the numerical analysis from Reference [141] (where the Higgs field was absent). Since in the present scenario the Higgs field during (most of the) inflation resides in its "false" vacuum, the significant impact of Higgs field dynamics will occur after end of inflation when the "inflaton" starts to generate the non-trivial Higgs symmetry breaking potential.

Author Contributions: Conceptualization: D.B., E.I.G., E.N., S.P.; Formal analysis: D.B., E.I.G., E.N., S.P.; Investigation: D.B., E.I.G., E.N., S.P. All authors have read and agreed to the published version of the manuscript.

Funding: This research has received partial support by COST Action CA-15117 (CANTATA) and COST Action CA-18108. D.B. thanks Ben-Gurion University of the Negev and Frankfurt Institute for Advanced Studies for generous support. E.N. and S.P. are partially supported by Bulgarian National Science Fund Grant DN 18/1.

Acknowledgments: The authors thank two of the referees for their constructive remarks which contributed to the improvement of the presentation.

Conflicts of Interest: The authors declare no conflict of interest

\section{References}

1. Guth, A.H. The Inflationary Universe: A Possible Solution to the Horizon and Flatness Problems. Phys. Rev. 1981, D23, 347-356. [CrossRef]

2. Starobinsky, A.A. Spectrum of relict gravitational radiation and the early state of the universe. JETP Lett. 1979, 30, 682-685.

3. Kazanas, D. Dynamics of the Universe and Spontaneous Symmetry Breaking. Astrophys. J. 1980, 241, L59-L63. [CrossRef]

4. Starobinsky, A.A. A New Type of Isotropic Cosmological Models Without Singularity. Phys. Lett. 1980, 91B, 99-102, [CrossRef]

5. Linde, A.D. A New Inflationary Universe Scenario: A Possible Solution of the Horizon, Flatness, Homogeneity, Isotropy and Primordial Monopole Problems. Phys. Lett. 1982, 108B, 389-393, [CrossRef]

6. Albrecht, A.; Steinhardt, P.J. Cosmology for Grand Unified Theories with Radiatively Induced Symmetry Breaking. Phys. Rev. Lett. 1982, 48, 1220-1223, [CrossRef]

7. Barrow, J.D.; Ottewill, A.C. The Stability of General Relativistic Cosmological Theory. J. Phys. 1983, A16, 2757. [CrossRef]

8. Blau, S.K.; Guendelman, E.I.; Guth, A.H. The Dynamics of False Vacuum Bubbles. Phys. Rev. 1987, D35, 1747. [CrossRef]

9. Kolb, E.; Turner, M. The Early Universe; Addison Wesley: Boston, MA, USA, 1990.

10. Linde, A. Particle Physics and Inflationary Cosmology; Harwood: Chur, Switzerland, 1990. 
11. Guth, A. The Inflationary Universe; Vintage, Random House: New York, NJ, USA, 1998.

12. Liddle, A.; Lyth, D. Cosmological Inflation and Large-Scale Structure; Cambridge Univ. Press: Cambridge, UK, 2000.

13. Dodelson, S. Modern Cosmology; Acad. Press: Cambridge, MA, USA, 2003.

14. Weinberg, S. Cosmology; Oxford Univ. Press: Oxford, UK, 2008.

15. Mukhanov, V. Physical Foundations of Cosmology; Cambride Univ. Press: Cambridge, UK, 2005.

16. Liddle, A.; Lyth, D. The Primordial Density Perturbations-Cosmology, Inflation and Origin of Structure; Cambridge Univ. Press: Cambridge, UK, 2009.

17. Lyth, D. Cosmology for Physicists; CRC: Boca Raton, FL, USA, 2017.

18. Calcagni, G. Classical and Quantum Cosmology; Springer: Berlin, Germany, 2017.

19. Gorbunov, D.; Rubakov, V. Introduction to the Theory of the Early Universe. Hot Big Bang Theory, 2nd ed.; World Scientific: Singapore, 2018.

20. Piattella, O. Lecture Notes in Cosmology; Springer: Berlin, Germany, 2018.

21. Benisty, D.; Guendelman, E.I. Inflation compactification from dynamical spacetime. Phys. Rev. 2018, D98, 043522. [CrossRef]

22. Liddle, A.R.; Lyth, D.H. COBE, gravitational waves, inflation and extended inflation. Phys. Lett. 1992, B291, 391-398. [CrossRef]

23. Liddle, A.R.; Lyth, D.H. The Cold dark matter density perturbation. Phys. Rept. 1993, 231, 1-105. [CrossRef]

24. Benisty, D.; Guendelman, E.I.; Saridakis, E.N. The Scale Factor Potential Approach to Inflation. arXiv 2019, arXiv:gr-qc/1909.01982.

25. Riess, A.G. Observational evidence from supernovae for an accelerating universe and a cosmological constant. Astron. J. 1998, 116, 1009-1038. [CrossRef]

26. Perlmutter, S. Measurements of $\Omega$ and $\Lambda$ from 42 high redshift supernovae. Astrophys. J. 1999, 517, 565-586. [CrossRef]

27. Riess, A.G. Type Ia supernova discoveries at $z>1$ from the Hubble Space Telescope: Evidence for past deceleration and constraints on dark energy evolution. Astrophys. J. 2004, 607, 665-687. [CrossRef]

28. Saridakis, E.N.; Bamba, K.; Myrzakulov, R.; Anagnostopoulos, F.K. Holographic dark energy through Tsallis entropy. JCAP 2018, 1812, 12. [CrossRef]

29. Vasak, D.; Kirsch, J.; Struckmeier, J. Dark energy and inflation invoked in CCGG by locally contorted space-time. arXiv 2019, arXiv:gr-qc/1910.01088.

30. Di Valentino, E.; Melchiorri, A.; Mena, O.; Vagnozzi, S. Non-minimal dark sector physics and cosmological tensions. Phys. Rev. 2020, D101, 063502. [CrossRef]

31. Perez, A.; Sudarsky, D.; Wilson-Ewing, E. Resolving the $H_{0}$ tension with diffusion. arXiv 2020, arXiv:astro-ph.CO/2001.07536.

32. Anagnostopoulos, F.K.; Basilakos, S. Constraining the dark energy models with $H(z)$ data: An approach independent of $H_{0}$. Phys. Rev. 2018, D97, 063503. [CrossRef]

33. Struckmeier, J.; Muench, J.; Vasak, D.; Kirsch, J.; Hanauske, M.; Stoecker, H. Canonical Transformation Path to Gauge Theories of Gravity. Phys. Rev. 2017, D95, 124048. [CrossRef]

34. Peebles, P.J.E.; Vilenkin, A. Quintessential inflation. Phys. Rev. 1999, D59, 063505. [CrossRef]

35. Nojiri, S.; Odintsov, S.D. Modified gravity with negative and positive powers of the curvature: Unification of the inflation and of the cosmic acceleration. Phys. Rev. 2003, D68, 123512. [CrossRef]

36. Cognola, G.; Elizalde, E.; Nojiri, S.; Odintsov, S.D.; Sebastiani, L.; Zerbini, S. A Class of viable modified $\mathrm{f}(\mathrm{R})$ gravities describing inflation and the onset of accelerated expansion. Phys. Rev. 2008, D77, 046009. [CrossRef]

37. De Laurentis, M.; Paolella, M.; Capozziello, S. Cosmological inflation in $F(R, G)$ gravity. Phys. Rev. 2015, D91, 083531. [CrossRef]

38. Chiba, T.; Okabe, T.; Yamaguchi, M. Kinetically driven quintessence. Phys. Rev. 2000, D62, 023511. [CrossRef]

39. Armendariz-Picon, C.; Mukhanov, V.F.; Steinhardt, P.J. A Dynamical solution to the problem of a small cosmological constant and late time cosmic acceleration. Phys. Rev. Lett. 2000, 85, 4438-4441. [CrossRef]

40. Armendariz-Picon, C.; Mukhanov, V.F.; Steinhardt, P.J. Essentials of k essence. Phys. Rev. 2001, D63, 103510. [CrossRef]

41. Chiba, T. Tracking K-essence. Phys. Rev. 2002, D66, 063514. [CrossRef] 
42. Saitou, R.; Nojiri, S. The unification of inflation and late-time acceleration in the frame of $k$-essence. Eur. Phys. J. 2011, C71, 1712. [CrossRef]

43. Wetterich, C. Variable gravity Universe. Phys. Rev. 2014, D89, 024005. [CrossRef]

44. Bueno Sanchez, J.C.; Dimopoulos, K. Trapped quintessential inflation in the context of flux compactifications. JCAP 2007, 710, 2. [CrossRef]

45. Bueno Sanchez, J.C.; Dimopoulos, K. Trapped Quintessential Inflation. Phys. Lett. 2006, B642, $294-301$. [CrossRef]

46. Dimopoulos, K.; Valle, J.W.F. Modeling quintessential inflation. Astropart. Phys. 2002, 18, 287-306. [CrossRef]

47. Bezrukov, F.L.; Shaposhnikov, M. The Standard Model Higgs boson as the inflaton. Phys. Lett. 2008, B659, 703-706. [CrossRef]

48. Choudhury, S.; Chakraborty, T.; Pal, S. Higgs inflation from new Kähler potential. Nucl. Phys. 2014, B880, 155-174. [CrossRef]

49. Dimopoulos, K.; Donaldson Wood, L.; Owen, C. Instant preheating in quintessential inflation with $\alpha$-attractors. Phys. Rev. 2018, D97, 063525. [CrossRef]

50. Dimopoulos, K.; Owen, C. Quintessential Inflation with $\alpha$-attractors. JCAP 2017, 1706, 027. [CrossRef]

51. Choudhury, S. COSMOS-e' - soft Higgsotic attractors. Eur. Phys. J. 2017, C77, 469. [CrossRef]

52. Gundhi, A.; Steinwachs, C.F. Scalaron-Higgs inflation. Nucl. Phys. 2020, B954, 114989. [CrossRef]

53. Ema, Y. Dynamical Emergence of Scalaron in Higgs Inflation. JCAP 2019, 1909, 27. [CrossRef]

54. Hossain, M.W.; Myrzakulov, R.; Sami, M.; Saridakis, E.N. Variable gravity: A suitable framework for quintessential inflation. Phys. Rev. 2014, D90, 023512. [CrossRef]

55. Hossain, M.W.; Myrzakulov, R.; Sami, M.; Saridakis, E.N. Class of quintessential inflation models with parameter space consistent with BICEP2. Phys. Rev. 2014, D89, 123513. [CrossRef]

56. Hossain, M.W.; Myrzakulov, R.; Sami, M.; Saridakis, E.N. Evading Lyth bound in models of quintessential inflation. Phys. Lett. 2014, B737, 191-195. [CrossRef]

57. Wali Hossain, M.; Myrzakulov, R.; Sami, M.; Saridakis, E.N. Unification of inflation and dark energy à la quintessential inflation. Int. J. Mod. Phys. 2015, D24, 1530014. [CrossRef]

58. Geng, C.Q.; Hossain, M.W.; Myrzakulov, R.; Sami, M.; Saridakis, E.N. Quintessential inflation with canonical and noncanonical scalar fields and Planck 2015 results. Phys. Rev. 2015, D92, 023522. [CrossRef]

59. Geng, C.Q.; Lee, C.C.; Sami, M.; Saridakis, E.N.; Starobinsky, A.A. Observational constraints on successful model of quintessential Inflation. JCAP 2017, 1706, 11. [CrossRef]

60. Kleidis, K.; Oikonomou, V. A Study of an Einstein Gauss-Bonnet Quintessential Inflationary Model. Nucl. Phys. B 2019, 948, 114765. [CrossRef]

61. Dimopoulos, K.; Donaldson-Wood, L. Warm quintessential inflation. Phys. Lett. 2019, B796, 26-31. [CrossRef]

62. Copeland, E.J.; Sami, M.; Tsujikawa, S. Dynamics of dark energy. Int. J. Mod. Phys. 2006, D15, $1753-1936$. [CrossRef]

63. Novikov, E.A. Quantum Modification of General Relativity. Electron. J. Theor. Phys. 2016, 13, 79-90.

64. Benitez, F.; Gambini, R.; Lehner, L.; Liebling, S.; Pullin, J. Critical collapse of a scalar field in semiclassical loop quantum gravity. arXiv 2020, arXiv:gr-qc/2002.04044.

65. Budge, L.; Campbell, J.M.; De Laurentis, G.; Keith Ellis, R.; Seth, S. The one-loop amplitude for Higgs +4 gluons with full mass effects. arXiv 2020, arXiv:hep-ph/2002.04018.

66. Bell, G.; Beneke, M.; Huber, T.; Li, X.Q. Two-loop non-leptonic penguin amplitude in QCD factorization. arXiv 2020, arXiv:hep-ph/2002.03262.

67. Fröhlich, J.; Knowles, A.; Schlein, B.; Sohinger, V. A path-integral analysis of interacting Bose gases and loop gases. arXiv 2020, arXiv:math-ph/2001.11714.

68. D'Ambrosio, F. Semi-Classical Holomorphic Transition Amplitudes in Covariant Loop Quantum Gravity. Ph.D. Thesis, Center for Theoretical Physics, Aix-Marseille University , Marseille, France, 2020.

69. Novikov, E.A. Ultralight gravitons with tiny electric dipole moment are seeping from the vacuum. Mod. Phys. Lett. 2016, A31, 1650092. [CrossRef]

70. Dekens, W.; Stoffer, P. Low-energy effective field theory below the electroweak scale: matching at one loop. JHEP 2019, 10, 197. [CrossRef]

71. Ma, C.T.; Pezzella, F. Stringy Effects at Low-Energy Limit and Double Field Theory. arXiv 2019, arXiv:hep-th/1909.00411. 
72. Jenkins, E.E.; Manohar, A.V.; Stoffer, P. Low-Energy Effective Field Theory below the Electroweak Scale: Operators and Matching. JHEP 2018, 3, 16. [CrossRef]

73. Brandyshev, P.E. Cosmological solutions in low-energy effective field theory for type IIA superstrings. Grav. Cosmol. 2017, 23, 15-19. [CrossRef]

74. Gomez, C.; Jimenez, R. Cosmology from Quantum Information. arXiv 2020, arXiv:hep-th/2002.04294.

75. Faraoni, V.; Capozziello, S. Beyond Einstein Gravity; Springer: Dordrecht, The Netherlands, 2011; Volume 170. [CrossRef]

76. Nojiri, S.; Odintsov, S.D.; Oikonomou, V.K. Modified Gravity Theories on a Nutshell: Inflation, Bounce and Late-time Evolution. Phys. Rep. 2017, 692, 1-104. [CrossRef]

77. Dimitrijevic, I.; Dragovich, B.; Koshelev, A.S.; Rakic, Z.; Stankovic, J. Cosmological Solutions of a Nonlocal Square Root Gravity. Phys. Lett. 2019, B797, 134848. [CrossRef]

78. Bilic, N.; Dimitrijevic, D.D.; Djordjevic, G.S.; Milosevic, M.; Stojanovic, M. Tachyon inflation in the holographic braneworld. JCAP 2019, 1908, 34. [CrossRef]

79. Odintsov, S.D.; Oikonomou, V.K. Geometric Inflation and Dark Energy with Axion F(R) Gravity. Phys. Rev. 2020, D101, 44009. [CrossRef]

80. Guendelman, E.I.; Kaganovich, A.B. The Principle of nongravitating vacuum energy and some of its consequences. Phys. Rev. 1996, D53, 7020-7025. [CrossRef] [PubMed]

81. Gronwald, F.; Muench, U.; Macias, A.; Hehl, F.W. Volume elements of space-time and a quartet of scalar fields. Phys. Rev. 1998, D58, 084021. [CrossRef]

82. Guendelman, E.I. Scale invariance, new inflation and decaying lambda terms. Mod. Phys. Lett. 1999, A14, 1043-1052. [CrossRef]

83. Guendelman, E.I.; Kaganovich, A.B. Dynamical measure and field theory models free of the cosmological constant problem. Phys. Rev. 1999, D60, 065004. [CrossRef]

84. Guendelman, E.I.; Kaganovich, A.B. Absence of the Fifth Force Problem in a Model with Spontaneously Broken Dilatation Symmetry. Ann. Phys. 2008, 323, 866-882. [CrossRef]

85. Guendelman, E.; Nissimov, E.; Pacheva, S.; Vasihoun, M. A New Mechanism of Dynamical Spontaneous Breaking of Supersymmetry. Bulg. J. Phys. 2014, 41, 123-129.

86. Guendelman, E.; Nissimov, E.; Pacheva, S. Vacuum structure and gravitational bags produced by metric-independent space-time volume-form dynamics. Int. J. Mod. Phys. 2015, A30, 1550133. [CrossRef]

87. Guendelman, E.; Nissimov, E.; Pacheva, S. Dark Energy and Dark Matter From Hidden Symmetry of Gravity Model with a Non-Riemannian Volume Form. Eur. Phys. J. 2015, C75, 472. [CrossRef]

88. Guendelman, E.; Nissimov, E.; Pacheva, S. Unified Dark Energy and Dust Dark Matter Dual to Quadratic Purely Kinetic K-Essence. Eur. Phys. J. 2016, C76, 90. [CrossRef]

89. Guendelman, E.; Nissimov, E.; Pacheva, S. Gravity-Assisted Emergent Higgs Mechanism in the Post-Inflationary Epoch; honorable mention in 2016 Gravity Research Foundation Competition for Essays on Gravitation. Int. J. Mod. Phys. 2016, D25, 1644008. [CrossRef]

90. Guendelman, E.; Nissimov, E.; Pacheva, S. Wheeler-DeWitt Quantization of Gravity Models of Unified Dark Energy and Dark Matter. Springer Proc. Math. Stat. 2017, 255, 99-113. [CrossRef]

91. Guendelman, E.; Nissimov, E.; Pacheva, S. Modified Gravity and Inflaton Assisted Dynamical Generation of Charge Confinement and Electroweak Symmetry Breaking in Cosmology. AIP Conf. Proc. 2019, 2075, 090030. [CrossRef]

92. Weinberg, S. Quantum Theory of Fields. Volume 2-Modern Applications; Cambridge Univ. Press: Cambridge, UK, 1996.

93. Kane, G. Modern Elementary Particle Physics. Explaining and Extending the Standard Model; Cambridge Univ. Press: Cambridge, UK, 2017.

94. Bekenstein, J.D. Gravitation and Spontaneous Symmetry Breaking. Found. Phys. 1986, 16, 409-422. [CrossRef]

95. Rubio, J. Higgs inflation. Front. Astron. Space Sci. 2019, 5, 50. [CrossRef]

96. Antoniadis, I.; Lykkas, A.; Tamvakis, K. Constant-roll in the Palatini- $R^{2}$ models. arXiv 2020, arXiv:gr-qc/2002.12681.

97. Ouseph, C.J.; Cheung, K. Higgs Inflation With Four-form Couplings. arXiv 2020, arXiv:hep-ph/2002.12010.

98. Ema, Y.; Mukaida, K.; van de Vis, J. Higgs Inflation as Nonlinear Sigma Model and Scalaron as its $\sigma$-meson. arXiv 2020, arXiv:hep-ph/2002.11739. 
99. Torabian, M. Electroweak vacuum stability and the Higgs field relaxation via gravitational effects. Class. Quant. Grav. 2020, 37, 65009. [CrossRef]

100. Adshead, P.; Pearce, L.; Shelton, J.; Weiner, Z.J. Stochastic evolution of scalar fields with continuous symmetries during inflation. arXiv 2020, arXiv:hep-ph/2002.07201.

101. Okada, N.; Raut, D.; Shafi, Q. SMART U(1) $X$ - Standard Model with Axion, Right handed neutrinos, Two Higgs doublets and $\mathrm{U}(1)_{X}$ gauge symmetry. arXiv 2020, arXiv:hep-ph/2002.07110.

102. Shaposhnikov, M.; Shkerin, A.; Zell, S. Quantum Effects in Palatini Higgs Inflation. arXiv 2020, arXiv:hep-ph/2002.07105.

103. Tenkanen, T.; Tomberg, E. Initial conditions for plateau inflation. arXiv 2020, arXiv:astro-ph.CO/2002.02420.

104. Shaposhnikov, M.; Shkerin, A.; Zell, S. Standard Model Meets Gravity: Electroweak Symmetry Breaking and Inflation. arXiv 2020, arXiv:hep-th/2001.09088.

105. Barrie, N.D.; Sugamoto, A.; Takeuchi, T.; Yamashita, K. Higgs Inflation, Vacuum Stability, and Leptogenesis. arXiv 2020, arXiv:hep-ph/2001.07032.

106. Sato, S.; Maeda, K.I. Stability of Hybrid Higgs Inflation. arXiv 2020, arXiv:gr-qc/2001.00154.

107. Passaglia, S.; Hu, W.; Motohashi, H. Primordial Black Holes as Dark Matter through Higgs Criticality. arXiv 2019, arXiv:astro-ph.CO/1912.02682.

108. Mishra, S.S.; Müller, D.; Toporensky, A.V. On generality of Starobinsky and Higgs inflation in the Jordan frame. arXiv 2019, arXiv:gr-qc/1912.01654.

109. Gialamas, I.D.; Lahanas, A.B. Reheating in $R^{2}$ Palatini inflationary models. arXiv 2019, arXiv:gr-qc/1911.11513.

110. Benisty, D. Inflation from Fermions. arXiv 2019, arXiv:gr-qc/1912.11124.

111. Einstein, A. Sitzungber. Preuss. Akad. Wiss. 23 ; Berlin-Brandenburgische Akademie der Wissenschaften: Berlin, Germany, 1923.

112. Einstein, A. Sitzungber. Preuss. Akad. Wiss. 137; Berlin-Brandenburgische Akademie der Wissenschaften: Berlin, Germany, 1923.

113. Eddington, A. The Mathematical Theory of Relativity; Univ. Press: Cambridge, UK, 1924.

114. Schrödinger, E. Space-Time Structure; Cambridge Univ. Press: Cambridge, UK, 1950.

115. Kijowski, J. Gravitation and spontaneous symmetry breaking. Gen. Rel. Grav. 1987, 9, 857. [CrossRef]

116. Ferraris, M.; Kijowski, J. On the Equivalence of the Relativistic Theories of Gravitation. Gen. Rel. Grav. 1982, 14, 165-180. [CrossRef]

117. Capozziello, S.; De Laurentis, M. Extended Theories of Gravity. Phys. Rep. 2011, 509, 167-321. [CrossRef]

118. Poplawski, N. Affine theory of gravitation. Gen. Rel. Grav. 2014, 46, 1625. [CrossRef]

119. Bejarano, C.; Delhom, A.; Jiménez-Cano, A.; Olmo, G.J.; Rubiera-Garcia, D. Geometric inequivalence of metric and Palatini formulations of General Relativity. Phys. Lett. 2020, B802, 135275. [CrossRef]

120. Delhom, A.; Olmo, G.J.; Orazi, E. Ricci-Based Gravity theories and their impact on Maxwell and nonlinear electromagnetic models. JHEP 2019, 11, 149. [CrossRef]

121. Nascimento, J.R.; Olmo, G.J.; Porfírio, P.J.; Petrov, A.Y.; Soares, A.R. Nonlinear $\sigma$-models in the Eddington-inspired Born-Infeld Gravity. arXiv 2019, arXiv:hep-th/1912.10779.

122. Delhom, A.; Macedo, C.F.B.; Olmo, G.J.; Crispino, L.C.B. Absorption by black hole remnants in metric-affine gravity. Phys. Rev. 2019, D100, 24016. [CrossRef]

123. Olmo, G.J.; Rubiera-Garcia, D.; Wojnar, A. Minimum main sequence mass in quadratic Palatini $f(R)$ gravity. Phys. Rev. 2019, D100, 44020. [CrossRef]

124. Harko, T.; Koivisto, T.S.; Lobo, F.S.N.; Olmo, G.J.; Rubiera-Garcia, D. Coupling matter in modified $Q$ gravity. Phys. Rev. 2018, D98, 84043. [CrossRef]

125. Olmo, G.J.; Rubiera-Garcia, D. Geons in Palatini Theories of Gravity. Fundam. Theor. Phys. 2017, 189, 161-190. [CrossRef]

126. Wojnar, A. Polytropic stars in Palatini gravity. Eur. Phys. J. 2019, C79, 51. [CrossRef]

127. Afonso, V.I.; Olmo, G.J.; Rubiera-Garcia, D. Scalar geons in Born-Infeld gravity. JCAP 2017, $1708,31$. [CrossRef]

128. Beltran Jimenez, J.; Heisenberg, L.; Olmo, G.J.; Rubiera-Garcia, D. Born-Infeld inspired modifications of gravity. Phys. Rep. 2018, 727, 1-129. [CrossRef]

129. Casana, R.; da Hora, E.; Rubiera-Garcia, D.; dos Santos, C. Topological vortices in generalized Born-Infeld-Higgs electrodynamics. Eur. Phys. J. 2015, C75, 380. [CrossRef] 
130. Odintsov, S.D.; Olmo, G.J.; Rubiera-Garcia, D. Born-Infeld gravity and its functional extensions. Phys. Rev. 2014, D90, 44003. [CrossRef]

131. Benisty, D.; Guendelman, E.I. Correspondence between the first and second order formalism by a metricity constraint. Phys. Rev. 2018, D98, 44023. [CrossRef]

132. Benisty, D.; Guendelman, E.I.; Vasak, D.; Struckmeier, J.; Stoecker, H. Quadratic curvature theories formulated as Covariant Canonical Gauge theories of Gravity. Phys. Rev. 2018, D98, 106021. [CrossRef]

133. Benisty, D.; Guendelman, E.I.; Struckmeier, J. Gauge theory of Gravity based on the correspondence between the 1st and the 2nd order formalisms. arXiv 2018, arXiv:hep-th/1808.01978.

134. Azri, H.; Nasri, S. Entropy Production in Affine Inflation. arXiv 2019, arXiv:gr-qc/1911.11495.

135. Spivak, M. Calculus on Manifolds-A Modern Approach to Classical Theorems of Advanced Calculus; CRC Press: Boca Raton, FL, USA, 2018; Chapter 5, p. 126,

136. Lim, E.A.; Sawicki, I.; Vikman, A. Dust of Dark Energy. JCAP 2010, 1005, 12. [CrossRef]

137. Henneaux, M.; Teitelboim, C. Quantization of Gauge Systems; Princeton Univ. Press: Princeton, NJ, USA, 1991.

138. Rothe, H.J.; Rothe, K.D. Classical and Quantum Dynamics of Constrained Hamiltonian Systems; World Scientific: Singapore, 2010.

139. Benisty, D.; Guendelman, E.; Nissimov, E.; Pacheva, S. Dynamically Generated Inflation from Non-Riemannian Volume Forms. Eur. Phys. J. 2019, C79, 806. [CrossRef]

140. Benisty, D.; Guendelman, E.I.; Nissimov, E.; Pacheva, S. Dynamically generated inflationary two-field potential via non-Riemannian volume forms. Nucl. Phys. 2020, B951, 114907. [CrossRef]

141. Guendelman, E.; Herrera, R.; Labrana, P.; Nissimov, E.; Pacheva, S. Emergent Cosmology, Inflation and Dark Energy. Gen. Rel. Grav. 2015, 47, 10. [CrossRef]

142. Ade, P.A.R. Planck 2013 results. XXII. Constraints on inflation. Astron. Astrophys. 2014, 571, A22. [CrossRef]

143. Adam, R. Planck intermediate results. XXX. The angular power spectrum of polarized dust emission at intermediate and high Galactic latitudes. Astron. Astrophys. 2016, 586, A133. [CrossRef]

144. Azri, H. Are there really conformal frames? Uniqueness of affine inflation. Int. J. Mod. Phys. 2018, D27, 1830006. [CrossRef]

145. Kijowski, J.; Werpachowski, R. Universality of affine formulation in general relativity theory. Rep. Math. Phys. 2007, 59, 1. [CrossRef]

(C) 2020 by the authors. Licensee MDPI, Basel, Switzerland. This article is an open access article distributed under the terms and conditions of the Creative Commons Attribution (CC BY) license (http:/ / creativecommons.org/licenses/by/4.0/). 